\title{
Adolf Hurwitz faltet Papier
}

\author{
Nicola M. R. Oswald
}

Eingegangen: 16. Juni 2014 / Angenommen: 8. August 2014 / Online publiziert: 24. Oktober 2014 (C) Springer-Verlag Berlin Heidelberg 2014

\section{Einleitung}

Es hat den Anschein, dass der berühmte Mathematiker Adolf Hurwitz (1859-1919) (Abb. 1) von eher ernsthaftem Charakter war. Kollegen beschrieben ihn stets als ,ruhig und besonnen“, gar „leidenschaftslos im Urteil“ ([10], S. XXIV) oder auch ,der stillen Denkarbeit gewidmet“ ([5], S. 168). Seine Frau Ida Samuel-Hurwitz charakterisierte ihn darüber hinaus als zurückhaltend und mit einer ,gewisse[n] Scheu“ vor Bekanntschaften ([8], S. 15). Mathematikhistorisch betrachtet, gehörte Adolf Hurwitz zu einer letzten Generation Universalgelehrter: Er erzielte herausragende Resultate in Funktionentheorie, Algebra, Zahlentheorie sowie Geometrie [6].

Überraschenderweise konnten wir herausfinden, dass dieser gewissenhafte Mathematiker ${ }^{1}$ seine Zeit auch mit Faltkonstruktionen aus Papier verbrachte. Im Folgenden werden wir einige Darstellungen untersuchen, die in Hurwitz' mathematischen Tagebüchern [7], verwahrt im Archiv der ETH Zürich², zu finden sind.

\section{Faltkonstruktionen in Hurwitz' mathematischen Tagebüchern}

„Hurwitz hat seit seiner Habilitation 1882 in ununterbrochenender Regelmäßigkeit von allem, was ihn wissenschaftlich beschäftigte, Aufzeichnungen gemacht und auf diese Weise eine Serie von 31 Tagebüchern hinterlassen, die ein getreues Bild seiner

\footnotetext{
${ }^{1}$ Eine biographische Schilderung von Hurwitz' Leben findet man in [11].

${ }^{2}$ Unter dem Verzeichnis HS 852:1-30
}

N. M. R. Oswald $(\bowtie)$

Institut für Mathematik, Universität Würzburg, Emil-Fischer-Straße 40, 97074 Würzburg, Deutschland

E-Mail: nicola.oswald@mathematik.uni-wuerzburg.de 
Abb. 1 Portrait von Adolf Hurwitz (1859-1919), Auszug aus Riesz' Register in Acta Mathematica von 1913 [13]
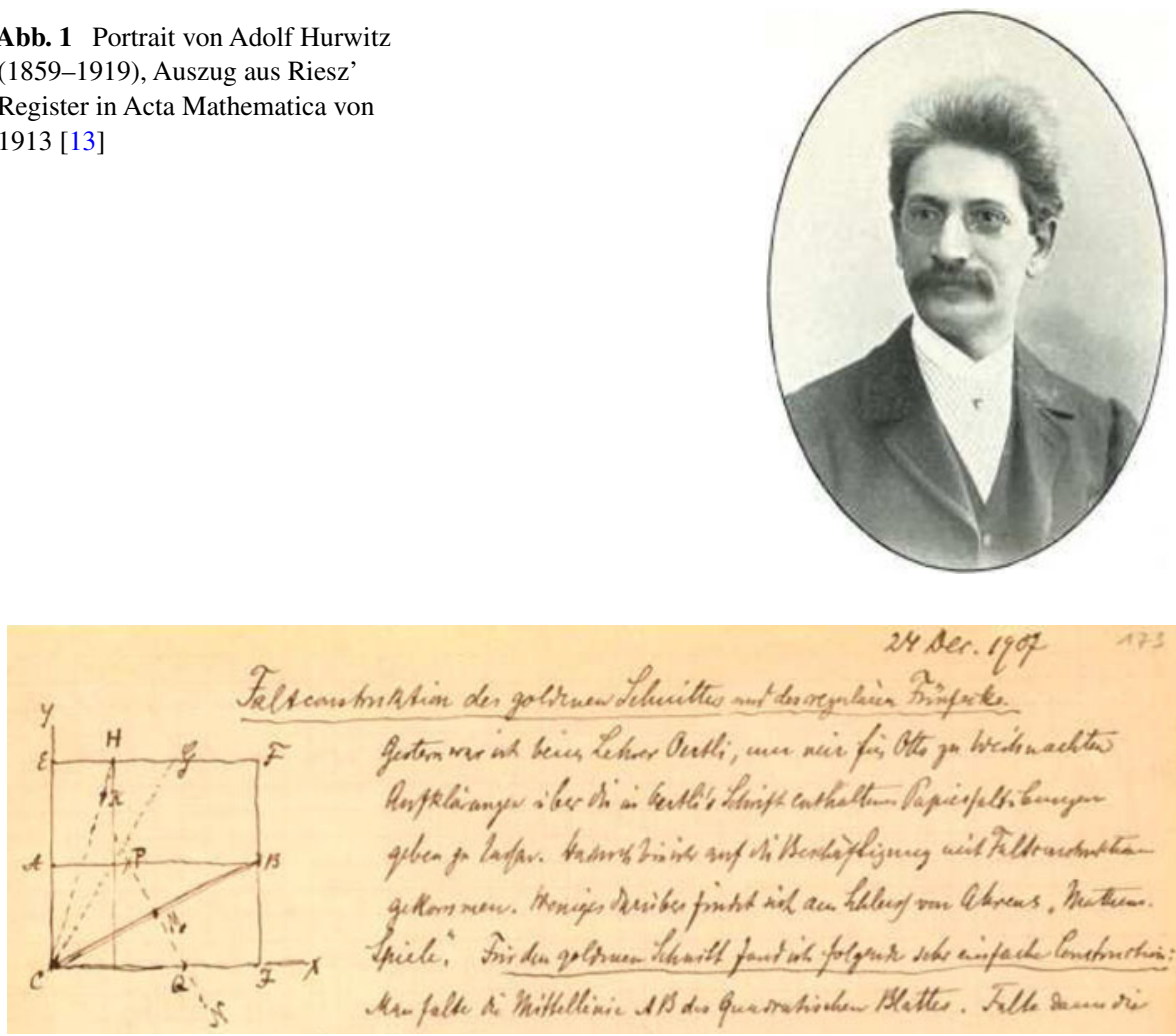

Abb. 2 Passage aus ([7], Nr. 22, S. 173)

beständig fortschreitenden Entwicklung geben und zugleich eine reiche Fundgrube für interessante und zur weiteren Bearbeitung geeignete Gedanken und Probleme sind“" ([5], S. 166).

Im 22. Tagebuch ${ }^{3}$ findet sich ein ungewöhnlicher Eintrag, der offensichtlich von Hurwitz' damals neunjährigem Sohn Otto angeregt worden war. Am 24. Dezember 1907 notierte Hurwitz unter dem Titel ,Faltconstruktion des goldenen Schnittes und des regulären Fünfecks. Gestern war ich beim Lehrer Oertli, um mir für Otto zu Weihnachten Aufklärung über die in Oertli's Schrift enthaltenen Papierfaltübungen geben zu laßen. Dadurch bin ich auf die Beschäftigung mit Faltconstruktionen gekommen“ (Abb. 2).

Hurwitz fuhr fort: „Weniges darüber findet sich am Schluß von Ahrens ,Mathematische Spiele“" . Für den goldenen Schnitt fand ich folgende sehr einfache Construction: Man falte die Mittellinie $A B$ des quadratischen Blattes. Falte dann die Diagonale $C B$, und die Halbierungslinie $C G$ des Winkels $E C B$ (indem man $C E$ auf $C B$ legt). Dann

\footnotetext{
${ }^{3}$ Vom 18. Dezember 1906 bis 22. Januar 1908

${ }^{4}$ Hier ist wahrscheinlich [2] gemeint, eine gekürzte Version von [1]. 


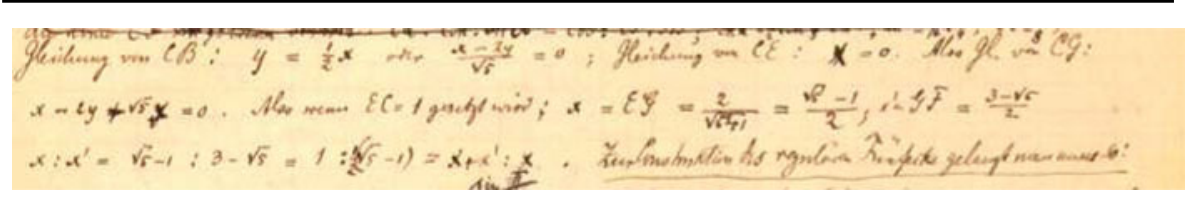

Abb. 3 „Also wenn $E C=1$ gesetzt wird; $x=E G=\frac{2}{\sqrt{5}+1}=\frac{\sqrt{5}-1}{2} ; x^{\prime}=G F=\frac{3-\sqrt{5}}{2} x: x^{\prime}=\sqrt{5}-$ $1: 3-\sqrt{5}=1: \frac{1}{2}(\sqrt{5}-1)=x+x^{\prime}: x$ (Im elften Satz im zweiten Buch der Elemente von Euklid ([3], B. II) befindet sich eine verwandte Konstruktion des goldenen Schnittes. Als Konstruktionsmethode für den Beweis von $a(a-x)=x^{2}$ werden hier statt der Winkelhalbierung Strecken abgetragen). Zur Construktion des regulären Fünfecks gelangt man nun so:" (Abb. 4)

Abb. 4 Die Idee hinter Hurwitz'

Fünfeck-Konstruktion

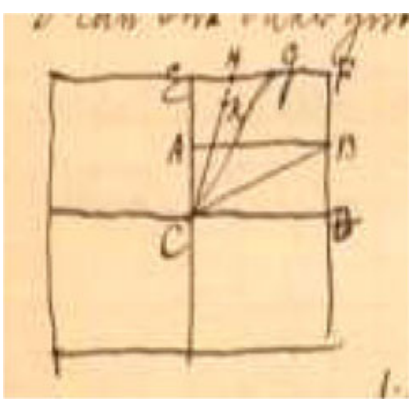

wird $E F$ im Punkte $G$ geteilt. “5 Mit Hilfe elementar-geometrischer Methoden bewies Hurwitz anschließend, dass das gefaltete Verhältnis tatsächlich dem goldenen Schnitt entspricht. Er stellte die „Gleichung von $C B: y=\frac{1}{2} x$ oder $\frac{x-2 y}{\sqrt{5}}=0$ “ auf, wobei er durch eine ,Normierung ' derselben durch den Faktor $\frac{2}{\sqrt{5}}$ eine darauf liegende Strecke der Länge 1 beschrieb. Dies entspricht der Länge von $C E$ für die Hurwitz die entsprechende „Gleichung von $C E: x=0$ “ aufstellte. Durch Summation der beiden bestimmt er anschließend die Winkelhalbierende $C G$ (siehe Abb. 3$)^{6}$

$$
\frac{x-2 y}{\sqrt{5}}+x=0 \Leftrightarrow x-2 y+\sqrt{5} x=x(\sqrt{5}+1)-2 y=0 .
$$

Diese einfache Papierfaltkonstruktion des goldenen Schnittes war Hurwitz' Ausgangspunkt für eine Konstruktion des regulären Fünfecks. Er arbeitete heraus, dass nach Halbierung von $E G$ in einem weiteren Punkt $H$ der folgende Zusammenhang gilt: $E H=\frac{1}{2} E G=\frac{\sqrt{5}-1}{4}=\sin \left(\frac{\pi}{10}\right)$, wobei $\frac{\pi}{10}$ offensichtlich dem Zentriwinkel eines regulären 20-Ecks entspricht.

Dadurch erhielt Hurwitz $\angle H C E=\frac{\pi}{10}$ beziehungsweise $\angle H C J=\frac{\pi}{2}-\frac{\pi}{10}=\frac{2 \pi}{5}$, was gleich dem Zentriwinkel eines regulären Fünfecks ist. Um darüber hinaus der Bedingung gleichlanger Seiten zu genügen, faltete Hurwitz $C J$ auf $C H$ und erhielt einen neuen Punkt $K$, webei die Länge von $C J$ gleich der Länge von $C K$ ist. Insgesamt

\footnotetext{
${ }^{5}$ Die aufmerksame Leserschaft ist an dieser Stelle dazu eingeladen, sich selbst an einem quadratischen Papier zu versuchen.

${ }^{6}$ Modern ausgedrückt entspricht dies der Normierung zweier Vektoren sowie der gewohnten Methode durch deren Summation die Winkelhalbierende zu bestimmen.
} 
Abb. 5 Illustration der vierten Regel
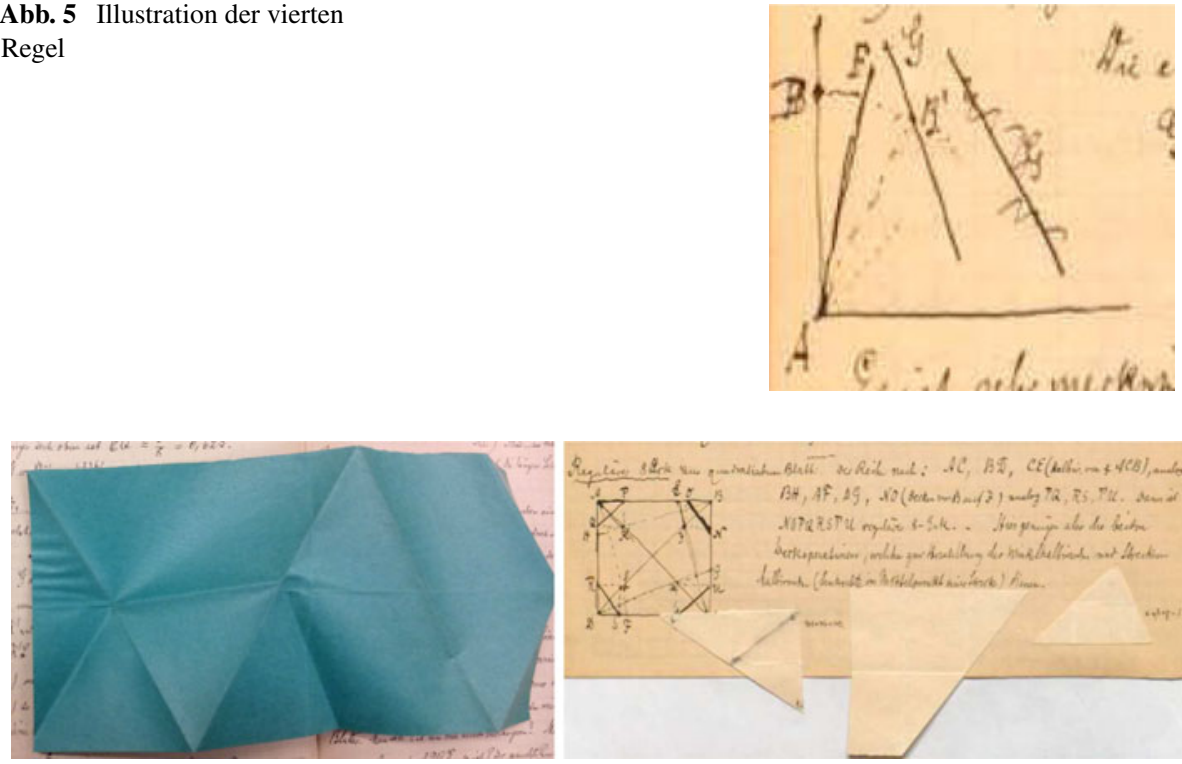

Abb. 6 Das linke Bild zeigt ein Stück Papier, das lose zwischen den letzten Seiten des 22. Tagebuchs aufgefunden wurde. Es kann nur angenommen werden, dass es Hurwitz selbst war, der die Faltlinien vornahm. Im rechten Bild ist eine Darstellung der regulären Achteck-Konstruktion zu sehen. Hier kann mit hoher Wahrscheinlichkeit davon ausgegangen werden, dass es sich bei den gefalteten Papierstücken um Originale handelt: Hurwitz hat diese sogar in seine Tagebuchseite eingeklebt

lässt sich damit das reguläre Fünfeck mit Mittelpunkt $C$ konstruieren, indem man um $C$ fünf Dreiecke der Art $K C J$ arrangiert.

Der Fortgang des Tagebucheintrags vermittelt den Eindruck, dass Hurwitz Gefallen an dem Gebiet des Papierfaltens gefunden hat. Er stellte ein Regelwerk für mögliche Konstruktionen auf, das wir im Folgenden skizzieren wollen:

„Bei praktischer Ausführung von Faltungen wird man bald beobachten, daß nur folgende Operationen mit Sicherheit auszuführen sind: 1.) Bestimmung des Durchschnitts zweier Faltlinien [...] 2.) Bestimmung der Senkrechten in der Mitte der Verbindungsgeraden zweier Punkte auf dieser Verbindungsgeraden. [...] 3.) Halbierung eines bekannten Winkels durch Auflegen des einen Schenkels auf den anderen. 4.) Auflegen eines Randpunktes $B$ auf eine Gerade $G$ bis die Faltlinie $A F$ durch einen anderen Randpunkt $A$ geht" (siehe Abb. 5).

Daran anschließend konstruierte Hurwitz ein reguläres Sechseck und ein gleichseitiges Dreieck aus einem rechteckigen Stück Papier und ein reguläres Achteck aus einem quadratischen Papier (siehe Abb 6).

Abgesehen von regulären Polygonen beschäftigte sich Adolf Hurwitz auch mit anderen Faltkonstruktionen ${ }^{7}$, wir wollen uns hier jedoch lediglich auf zwei weitere seiner Ideen bezüglich des Fünfecks konzentrieren. Es gibt recht unterschiedliche

\footnotetext{
${ }^{7}$ Tatsächlich haben Faltkonstruktionen Vorteile gegenüber der Konstruktion mit Zirkel und Lineal; so können etwa die Winkeldreiteilung (siehe [9], Kap. 10) und die Zahl $\sqrt[3]{2}$ (siehe [14], Kap. 8.4) durch Papierfalten konstruiert werden.
} 


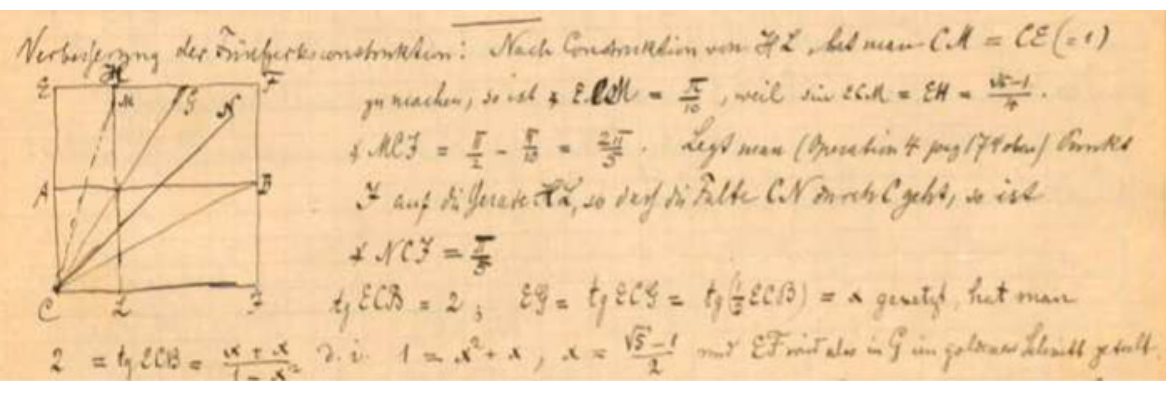

Abb. 7 ,Nach Construktion von $H L$, hat $\operatorname{man} C M=C E(=1)$ zu machen, so ist $\angle E C M=\frac{\pi}{10}$, weil $\sin E C M=E H=\frac{\sqrt{5}-1}{4} . \angle M C J=\frac{\pi}{2}-\frac{\pi}{10}=\frac{2 \pi}{5}$. Legt man (Operation 4 pag 174 oben) Punkt $J$ auf die Gerade $H L$, so daß die Falte $C N$ durch $C$ geht, so ist $\angle N C J=\frac{\pi}{5}$

Wege, ein solches zu falten, und Hurwitz' erster Ansatz ließ sicherlich noch Platz für Vereinfachungen. Tatsächlich findet man auf der nächsten Seite seines mathematischen Tagebuchs einen Eintrag mit dem Titel „Verbesserung der Fünfecksconstruction“:

$\operatorname{tg} E C B=2, E G=\operatorname{tg} E C G=\operatorname{tg}\left(\frac{1}{2} E C B\right)=\alpha$ gesetzt, hat man $2=\operatorname{tg} E C B=$ $\frac{x+x}{1-x^{2}}$ d. i. $1=x^{2}+x, x=\frac{\sqrt{5}-1}{2}$ und $E F$ wird also in $G$ im goldenen Schnitt geteilt" ${ }^{* 8}$ (Abb. 7).

Eine Art Hauptresultat von Hurwitz lässt sich schließlich am Ende des Tagebucheintrags ausmachen, wo er eine weitere Vereinfachung der Konstruktion eines regulären Fünfecks aus einem rechtwinkligen Stück Papier angibt: „In dem rechtwinkligen Blatt (Seite $1: 2$ ) $A B C D$ faltet man zunächst die Linien $a_{1}$ und $a a$. Sodann die beiden Linien $b$, die Linien $c$, welche die Winkel zwischen $a_{1}$ und $b$ halbieren, die Linien $d$, dann Punkt $B$ auf $d$ gelegt, giebt Linie $e=O P$, analog entsteht $O S$; Punkt $O$ auf $S$ gelegt giebt $q$ und $Q$ (als Durchschnitt von $q$ und $d$ ), analog $O$ auf $P$ gelegt giebt $R$. Dann ist $O P Q R S$ ein reguläres 5-Eck mit dem Mittelpunkt $M$. Um ein reguläres Fünfeck mit fertigen Seiten zu erhalten, kann man die Streckenhalbierenden zu $O M$, $P M, Q M, R M, S M$ falten“ (Abb. 8).

Es ist bemerkenswert, dass Hurwitz die Idee des Papierfaltens und damit verbundene Anwendungen offensichtlich sehr ernsthaft behandelte. In seinem Tagebucheintrag war es ihm ein Anliegen, exakte Anleitungen für die Konstruktionen des regulären Fünfecks anzugeben. Dies ist dadurch hervorgehoben, dass es bei seiner letzten Skizze (Abb. 8) so aussieht, als ob er die Anleitung zunächst mit Bleistift vorgeschrieben und anschließend ordentlich mit Füller nachgefahren hat. Im Anschluss gab er zusätzlich einen Beweis an: „Zum Beweis dieser Construction betrachte man ein reguläres 5-Eck $O P Q R S$ und setze die Diagonale $S P=1$. Damit ist $\angle P O B=\frac{\pi}{5}=\angle P O Q=\angle Q O R=\angle R O S$. Daher $\angle Q O M=\frac{\pi}{10}$ und $Q$ liegt auf der Geraden $d$, welche das Stück $\frac{\sqrt{5}-1}{4}=\sin \frac{\pi}{10}$ von $E C$ abschneidet. Da außerdem $Q$ senkrecht über der Mitte von $O S$ liegt, so leuchtet die Richtigkeit der Construction ein.“

\footnotetext{
${ }^{8}$ tg steht für Tangens.
} 


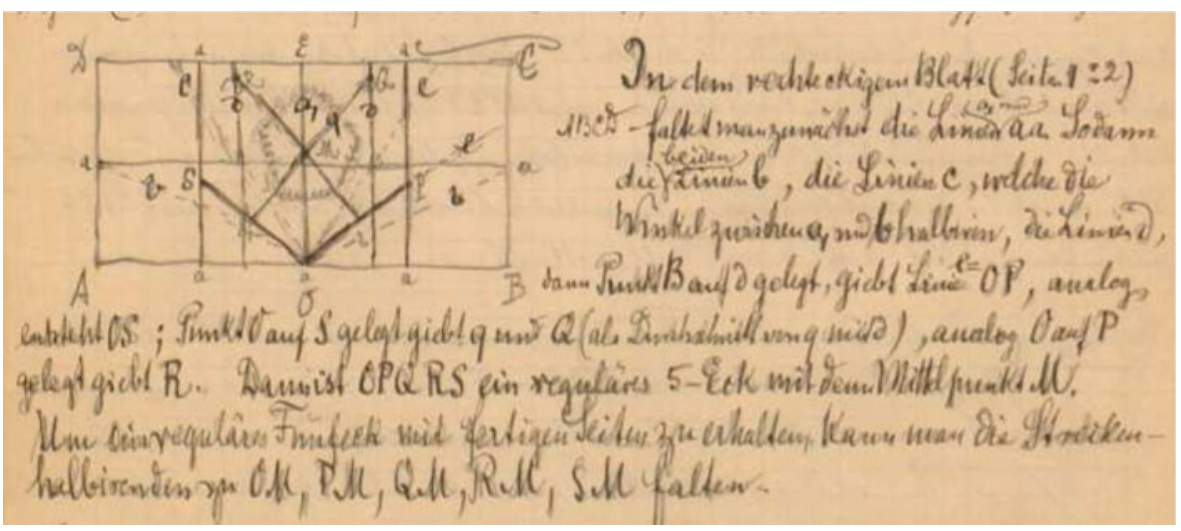

Abb. 8 Passage aus ([7], No. 22, S. 176)

Abb. 9 Fundstück aus dem 24.

Tagebuch (vom 19. Februar 1910 bis 26. Oktober 1911)

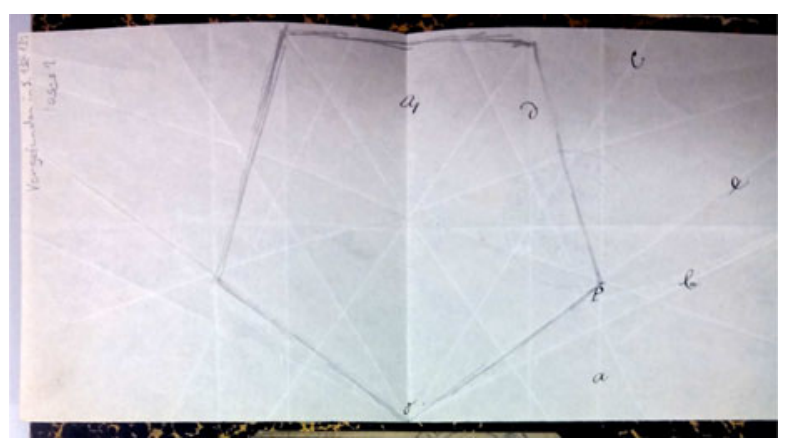

Dass diese letzte Konstruktion absolut nicht trivial war, wird durch ein loses Blatt illustruiert, welches zwei Tagebücher später unerwartet aufgefunden wurde. Die Handschrift der kleinen Buchstaben deutet darauf hin, dass es tatsächlich Hurwitz war, der dieses Fünfeck faltete (siehe Abb. 9).

\section{Epilog: Wie das Papierfalten zu Hurwitz kam}

Hurwitz' anfängliche Bemerkung über Ottos Lehrer Oertli deutet bereits darauf hin, dass Papierfalten eine gewisse Rolle in der mathematischen Erziehung von Kindern zu dieser Zeit spielte. Tatsächlich lässt sich diese pädagogische Herangehensweise zurückverfolgen auf Friedrich Fröbel (1782-1852), bekannt als Begründer der ,Kindergarten“-Bewegung und Erfinder der so genannten ,Fröbel-Gaben“9 . Er ,,[...] erkannte schon frühzeitig [...] unter anderem die große Nützlichkeit der kindlichen

\footnotetext{
${ }^{9}$ Eine Ansammlung pädagogischer Spielsachen, von denen eine im Folgenden näher beschrieben werden wird. Im Englischen 'kindergarten gifts' genannt.
} 
Beschäftigung mit dem Papierfalten und Flechten"[4]. ${ }^{10}$ Sein weitreichender Einfluss bezüglich der Faltübungen zur mathematischen Erziehung zeigt sich in [15], einem indischen Lehrbuch. Interessanterweise schreibt Sundara Row ${ }^{11}$ in seiner Einleitung: „Die Idee zu diesem Buch wurde angeregt durch meine Kindergarten-Gabe No. VIII - Papierfalten. Die Gabe beinhaltet 200 verschiedenfarbige Papierquadrate, eine Mappe, und Diagramme und Anleitungen für Faltungen. "12 Anschließend daran ging der indisch-deutsche Austausch sogar weiter: Rows Buch war dem deutschen Mathematiker und Experten für Unterhaltungsmathematik Wilhelm Ahrens (1872-1927) wohlbekannt. In seiner zweibändigen Sammlung, Mathematische Unterhaltungen und Spiele ([1], Kp. XXIII) von 1901 widmete er ein ganzes Kapitel den Konstruktionen durch Papierfalten und bemerkte vorab: „In diesem Kapitel beabsichtigen wir, einige Proben aus einem von dem indischen Mathematiker Sundara Row 1893 herausgegebenen Buche zu geben, in dem gezeigt wird, wie man zu verfahren hat, wenn man die geometrische Konstruktion lediglich durch Falten von Papier ausführen will [...]“. Damit setzte Ahrens Rows Anliegen fort, ,,nicht nur eine Unterstützung beim Unterricht der Geometrie in Schulen und Hochschulen zu bieten, sondern auch mathematische Unterhaltung für Jung und Alt zu ermöglichen [...]"13 ([15], S. vi), ein Anliegen, welches bei Adolf Hurwitz augenscheinlich Erfolg hatte.

\section{Literatur}

1. Ahrens, W.: Mathematische Unterhaltungen und Spiele. B. G. Teubner, Leipzig (1901)

2. Ahrens, W.: Mathematische Spiele. B. G. Teubner, Leipzig (1907)

3. Euklid.: Die Elemente, Bücher I-XIII. Clemens Thaer, Thun, reprint, 2nd edn (1996)

4. Flachsmeyer, J.: Origami und Mathematik. Heldermann Verlag, Berlin (2008)

5. Hilbert, D.: Adolf Hurwitz. Math. Annalen. 83, 161-168 (1921)

6. Hurwitz, A.: Mathematische Werke. Bd. 1. Funktionentheorie; Bd. 2. Zahlentheorie, Algebra und Geometrie. Birkhäuser, Basel (1932)

7. Hurwitz, A.: Die Mathematischen Tagebücher und der übrige handschriftliche Nachlass von Adolf Hurwitz. Zurich, ETH Library. HS. 582, 1-30. http://www.e-manuscripta.ch/ (1985)

8. Hurwitz-Samuel, I.: Erinnerungen an die Familie Hurwitz, mit Biographie ihres Gatten Adolph Hurwitz, Prof. f. höhere Mathematik an der ETH. Zürich, ETH Library. HS. 583a, 2 (1984)

9. Martin, G.: Geometric Constructions. Springer, Heidelberg (1998)

10. Meissner, E.: Gedächtnisrede auf Adolf Hurwitz, gehalten am 21. November 1919 im Krematorium Zürich. Vierteljahrsschrift der Naturforschenden Gesellschaft in Zürich. 64, 855-857 (1919)

\footnotetext{
${ }^{10}$ Einen Hinweis darauf, dass Fröbels Ideen nachhaltigen Einfluss hatten, lässt sich in ([16], S. A 135) nachlesen: ,Wer freilich von der vorgefaßten Meinung über die Mathematik ausgeht [ . .] wird es befremdlich finden, daß sie schon in Spielen des kleinen Kindes wie Stäbchenlegen, Papierfalten und Ausschneiden [...] ihren Ausruck findet."

${ }^{11}$ Manchmal auch als ,Rao“ anstatt ,Row“ zitiert

12 ,The idea of this book was suggested to me by Kindergarten Gift No. VIII - Paper-folding. The gift consists of 200 variously coloured squares of paper, a folder, and diagrams and instructions for folding." Wahrscheinlich wurde Row mit den ,Fröbel-Gaben“ durch Pandita Ramabai Sarawati bekannt gemacht, einer indischen Aktivistin der Frauenrechtsbewegung und Pionierin im Bildungswesen. Während ihres Aufenthalts in den USA von 1886 bis 1889 wurde sie Anhängerin von Fröbels Erziehungsmethoden (siehe [12], S. 145).

13 ,not only to aid the teaching of Geometry in school and colleges, but also to afford mathematical recreation to young and old [...]“
} 
11. Oswald, N., Steuding, J.: Complex continued fractions - early work of the brothers Adolf and Julius Hurwitz. Arch. Hist. Exact Sci. 68, 499-528 (2014)

12. Rambai, P.: Pandita Ramabai's American Encounter: The Peoples of the United States (1899) . Translated and edited by Meera Kosambi. Indian University Press, Bloomington (2003)

13. Riesz, M.: Acta mathematica, 1882-1912. Table générale des tomes 1-35. Almqvist and Wiksells, Uppsala (1913)

14. Steuding, J., Oswald, N.: Elementare Zahlentheorie. Ein sanfter Einstieg in die höhere Mathematik. Springer, Heidelberg (2014)

15. Sundara Row, T.: Geometrical exercises in paper folding. Addison Co, Madras (1893)

16. Timerding, H.: Die Mathematischen Wissenschaften. Die Verbreitung mathematischen Wissens und mathematischer Auffassung. B. G. Teubner, Leipzig (1914) 